\title{
Male Circumcision
}

National Cancer Institute

\section{Source}

National Cancer Institute. Male Circumcision. NCI Thesaurus. Code C87068.

Surgical removal of the penile foreskin. 